\title{
La Resolución 1325 del Consejo de Seguridad de las Naciones Unidas. A 15 años de su adopción ${ }^{1}$
}

\author{
Security Council Resolution 1325. Fifteen Years after its Adoption
}

\section{Ximena Gauché Marchetti}

xgauche@udec.cl

Doctora en Derecho, Universidad Autónoma de Madrid. Master Interuniversitario en Diplomacia y Relaciones Internacionales, Universidad Complutense de Madrid. Profesora Asociada de la Facultad de Ciencias Jurídicas y Sociales de la Universidad de Concepción. Directora del Programa Interdisciplinario sobre Protección Integral de la Infancia y la Adolescencia, y Coordinadora del Grupo Interdisciplinario de Investigación en Derechos Humanos y Democracia, de la misma universidad.

Resumen: Con la adopción de la Resolución 1325 por el Consejo de Seguridad de las Naciones Unidas en el año 2000, se dio inicio a una etapa en que una agenda institucional sobre igualdad de las mujeres en el ámbito de la paz y la seguridad internacional ha quedado instalada en la comunidad mundial. En ese contexto, este artículo, de tipo descriptivo, tiene como propósito identificar y comunicar el contenido de esta agenda sobre mujeres, paz y seguridad, y conocer su revisión institucional en el año 2015, oportunidad en que cumplió quince años y se conmemoraron setenta años de la organización.

Palabras claves: mujeres, género, Consejo de Seguridad.

Abstract: With the adoption in 2000 of Resolution 1325 by the United Nations Security Council, an agenda on women, peace and international security was installed. In this context, this article is descriptive and has the purpose to identify and communicate the content of this agenda on women, peace and security, checking its revision in 2015, the year of its fifteenth anniversary and the 70 years of the United Nations.

Keywords: women, gender, Security Council.

1 Artículo recibido el 11.03.2016 y aceptado el 06.06.2016.

Este artículo es parte del Proyecto de Investigación "Resoluciones del Consejo de Seguridad de Naciones Unidas sobre mujeres, paz y seguridad. Planes de Acción Nacional y otras estrategias para su implementación, y propuestas para la mayor participación de mujeres en el ámbito de seguridad y defensa en chile", financiado por la Academia Nacional de Estudios Políticos y Estratégicos (ANEPE) y convocado por su Departamento de Investigación, Código 201511. 


\section{La adopción de la Resolución 1325 y sucesoras: La respuesta institucional del Consejo de Seguridad de Naciones Unidas a la histórica exclusión de las mujeres en los ámbitos de la paz, defensa y seguridad internacionales}

Considerando que el principal propósito de la Organización de Naciones Unidas (ONU) es el mantenimiento de la paz y la seguridad internacionales, la violencia dirigida contra mujeres en diversos conflictos bélicos, visibilizada desde la segunda mitad del siglo XX, hizo que el tema de protegerlas especialmente fuera entrando en la agenda de la paz y seguridad mundial teniendo presente que antes "el silencio era ensordecedor, a pesar de la espantosa realidad de esa práctica en todos los conflictos armados"2.

Si bien se ha reconocido que la violencia sexual afecta tanto a hombres como a mujeres en circunstancias extremas, se ha tomado conciencia que las mujeres son más vulnerables a padecerla y además en ellas las consecuencias, desde el punto de vista de su rol en el proceso reproductivo, por ejemplo, pueden ser más críticas ${ }^{3}$. Además, no es sólo la violencia la forma en que las mujeres son víctimas de desigualdad en el ámbito de la seguridad internacional. También se manifiesta ésta en la menor presencia en los procesos de paz, formales o informales; en la intervención en la configuración y despliegue de operaciones de paz; o en fenómenos como el desplazamiento o refugio, la trata, la esclavitud sexual o prácticas como el matrimonio forzado, de frecuente uso por Boko Haram ${ }^{4}$ y antes en el conflicto de Sierra Leona de inicio de la década del 2000, por ejemplo ${ }^{5}$.

A partir de estas constataciones y teniendo presente la transversalidad de género que se asume por la ONU como una estrategia para alcanzar la meta de la igualdad de sexos - impulsada por la Conferencia de Beiging en 1995 y remarcada luego en los Objetivos de Desarrollo del Milenio en 2000 - se han ido elaborando planes de acción y estrategias para incorporar el tema

2 Judith Gardan, "La mujer, los derechos humanos y el derecho internacional humanitario", Revista Internacional de la Cruz Roja 147 (1998): 453-467.

3 Women 2000, "Sexual Violence and Armed Conflict: United Nations Response", Published to Promote the Goals of the Beijing Declaration and the Platform for Action, United Nations, Division for the Advancement of Women, Department of Economic and Social Affairs, http://www.un.org/womenwatch/daw/public/w2apr98.htm (consultado el 20 de junio de 2016).

4 Informe S/2015/716 del Secretario General de las Naciones Unidas, 16 de septiembre de 2015, párrafo 87.

5 En el marco de su trabajo y por vez primera en la historia, el Tribunal para Sierra Leona, creado por Resolución 1315 del Consejo de Seguridad, dictaminó que los matrimonios forzados constituyen un crimen contra la humanidad dedicando sendos trabajos jurisprudenciales a dar por configurada así en el ámbito penal internacional una de las peores formas de discriminación a las mujeres. Un completo estudio sobre los antecedentes de este conflicto y los pronunciamientos jurisdiccionales en la materia se encuentra en Amy, Palmer "An Evolutionary Analysis of Gender-Based War Crimes and the Continued Tolerance of "Forced Marriage"”, Northwestern Journal of International Human Rights 7(1) (2009): 143-159. 
Revista Tribuna Internacional

Volumen $5 \cdot \mathrm{N}^{\text {o }} 9 \cdot 2016 \cdot$ pp. 53-68

ISSN 0719-482X (versión en línea)

de las mujeres en sus más variados ámbitos de competencia, llegando así al mantenimiento de la paz y la seguridad internacional ${ }^{6}$.

De esta manera es que el Consejo de Seguridad de Naciones Unidas (CSNU) debió entrar en esta dinámica ${ }^{7}$. Lo hizo decididamente con su Resolución 1325, del año 2000, instalando el tema en la agenda internacional ${ }^{8}$.

Antes en todo caso, este Consejo había dado algunos pasos en resoluciones de los años 1999 y 2000. Incluso en los inicios de la década de los 90 y desde que en el medio del conflicto de los Balcanes se considerara a la violencia sexual en diversas formas como una nueva y sistemática forma de limpieza étnica ya manifestaba su consternación por las informaciones acerca de "las detenciones y violaciones sistemáticas, masivas y organizadas de mujeres, en particular mujeres musulmanas, en Bosnia y Herzegovina"'. En efecto, la violencia sexual desplegada en el conflicto de los Balcanes puso una voz de alerta institucional contra toda violencia que se produjera en el contexto de un conflicto, incluida la sexual ${ }^{10}$.

Para 1999 y a través de la Resolución 1261 instó a las partes que estuvieran en conflicto a tomar medidas especiales para proteger a los niños, y en particular a las niñas, de la violación y otras formas de abuso sexual y de la violencia basada en el género en situaciones de conflicto armado, y a tener presente las necesidades especiales de las niñas durante estos conflictos y

6 Esto se ha dado a la par de la adopción de un extenso marco normativo que se ha ido desarrollando con los años, a partir de la Convención para la Eliminación de toda forma de discriminación contra la mujer o CEDAW, de 1979, y a la Convención Interamericana para prevenir, sancionar y erradicar la violencia contra la mujer, conocida como Convención de Belén de Pará, de 1994. Un interesante trabajo sobre la incorporación de género en el ámbito internacional se encuentra en Hilary, Charlesworth, "Not Waving but Drowning: Gender Mainstreaming and Human Rights in the United Nations", Harvard Human Rights Journal 18 (2005).

7 Un par de décadas antes, en 1974 y en plena Guerra Fría, la Asamblea General aprobó en sesión de 14 de diciembre, la Resolución 3318 (XXIX) que contenía la Declaración sobre la Protección de la Mujer y el Niño en estados de emergencia o de conflicto armado que ya en esa época denotaba la preocupación por la cuestión, aunque su tenor muestra que no había consciencia aún sobre los distintos roles que juegan las mujeres en el marco de los conflictos - quienes no sólo pueden ser vistas como víctimas - ni tampoco había noción clara del diferente rol que ocupan hombres y mujeres en la sociedad.

8 Esto se ha hecho en el marco a su vez del artículo 25 de la Carta de las Naciones Unidas por el que sus miembros han convenido en “....aceptar y cumplir las decisiones del Consejo de Seguridad de acuerdo con esta Carta”.

9 Resolución S/RES/798 del Consejo de Seguridad de las Naciones Unidas, 18 de diciembre de 1992.

10 La Declaración y Plataforma de Acción de Viena de 1993 señala que "Las violaciones de los derechos humanos de la mujer en situaciones de conflicto armado constituyen violaciones de los principios fundamentales de los derechos humanos y el derecho humanitario internacionales. Todos los delitos de ese tipo, en particular los asesinatos, las violaciones sistemáticas, la esclavitud sexual y los embarazos forzados, requieren una respuesta especialmente eficaz". Declaración y Programa de Acción de Viena, A/CONF.157/23, 25 de mayo de 1993, Párrafo 38, Capítulo II. Por su parte, la Declaración y Plataforma de Beijing de 1995 indica que: "Aunque hay comunidades enteras que sufren las consecuencias de los conflictos armados y del terrorismo, las mujeres y las niñas se ven particularmente afectadas a causa de su condición en la sociedad y de su sexo. Las partes en los conflictos a menudo violan a las mujeres con impunidad, utilizando a veces la violación sistemática como táctica de guerra y de terrorismo. Los efectos de la violencia contra la mujer y de la violación de los derechos humanos de la mujer en tales situaciones son experimentados por mujeres de todas las edades, que sufren desplazamientos, pérdida del hogar y de los bienes, pérdida o desaparición involuntaria de parientes cercanos, pobreza y separación y desintegración de la familia y que son víctimas de actos de asesinato, terrorismo, torturas, desapariciones involuntarias, esclavitud sexual, violaciones, abusos sexuales y embarazos forzados en situaciones de conflicto armado, especialmente como resultado de políticas de depuración étnica y otras formas de violencia nuevas e incipientes. Ello se ve agravado por las traumáticas consecuencias de carácter social, económico y psicológico causadas por los conflictos armados y la ocupación y dominación extranjeras, consecuencias que se sufren durante toda la vida". Informe A/CONF.177/20 de la Cuarta Conferencia Mundial sobre la Mujer, 17 de octubre de 1995, Párrafo 135. 
La Resolución 1325 del Consejo de Seguridad de las Naciones Unidas. A 15 años de su adopción Ximena Gauché Marchetti

después de ellos, en especial en la prestación de asistencia humanitaria ${ }^{11}$. El mismo año, con la Resolución 1265, reconoció los efectos directos y específicos de los conflictos armados en la mujer, manifestando la acogida del órgano a la incorporación de la perspectiva de género en la asistencia humanitaria y la violencia contra la mujer, observando la importancia de incluir en los mandatos de las operaciones de paz, mantenimiento y consolidación de la paz, disposiciones especiales para mujeres y niños, y llamando al Secretario General a velar porque el personal de Naciones Unidas tenga formación en temas de género ${ }^{12}$.

En el año 2000, en tanto, el CSNU reafirmó con la Resolución 1296 su preocupación por los efectos de los conflictos en la población civil, especialmente mujeres, niños y otros grupos vulnerables $^{13}$, mientras que en la Resolución 1314, relativa a los niños y los conflictos armados, subrayó la necesidad de tener en cuenta las necesidades y vulnerabilidades específicas de las niñas ${ }^{14}$.

Sin perjuicio de estas prematuras referencias, como ya se apuntó fue a partir de la Resolución 1325 del mismo año 2000 que se asumió el cambio de paradigma y el carácter imperante del tema en la agenda mundial ${ }^{15}$, siendo así validado en varios contextos ${ }^{16}$.

En cuanto a su contenido, junto con reconocer que dentro de los civiles, son las mujeres y niños las mayores víctimas de los conflictos, la Resolución 1325 reconoce el rol que le cabe a ellas en la prevención y solución de los conflictos y en la consolidación de la paz. También subraya la importancia de que participen en pie de igualdad e intervengan plenamente en todas las iniciativas encaminadas al mantenimiento y el fomento de la paz y la seguridad, y la necesidad de aumentar su participación en los procesos de adopción de decisiones en materia de prevención y solución de conflictos. Reconoce además la urgente necesidad de incorporar una perspectiva de género en las operaciones de mantenimiento de la paz. Busca entonces la incorporación integral de ella en todos los aspectos que suponen el mantenimiento y fomento de la paz y la seguridad internacionales, involucrando directamente en este propósito al

\footnotetext{
Resolución S/RES/1261 del Consejo de Seguridad de las Naciones Unidas, 30 de agosto de 1999.

Resolución S/RES/1265 del Consejo de Seguridad de las Naciones Unidas, 17 de septiembre de 1999.

Resolución S/RES/1296 del Consejo de Seguridad de las Naciones Unidas, 19 de abril de 2000.

Resolución S/RES/1314 del Consejo de Seguridad de las Naciones Unidas, 11 de agosto de 2000.

Resolución S/RES/1325 del Consejo de Seguridad de las Naciones Unidas, 31 de octubre de 2000.

6 Por ejemplo, el Preámbulo del Protocolo Adicional a la Carta Africana de Derechos Humanos relativo a los Derechos de la Mujer hace referencia expresa a la Resolución 1325 del CSNU y trata en detalle la situación de la mujer en conflicto armado en su artículo 11. Artículo 11 Protocolo Adicional a la Carta Africana de Derechos Humanos relativo a los Derechos de la Mujer. "Protection of Women in Armed Conflicts. 1. States Parties undertake to respect and ensure respect for the rules of international humanitarian law applicable in armed conflict situations, which affect the population, particularly women. 2. States Parties shall, in accordance with the obligations incumbent upon them under international humanitarian law, protect civilians including women, irrespective of the population to which they belong, in the event of armed conflict. 3. States Parties undertake to protect asylum seeking women, refugees, returnees and internally displaced persons, against all forms of violence, rape and other forms of sexual exploitation, and to ensure that such acts are considered war crimes, genocide and/or crimes against humanity and that their perpetrators are brought to justice before a competent criminal jurisdiction. 4. States Parties shall take all necessary measures to ensure that no child, especially girls under 18 years of age, take a direct part in hostilities and that no child is recruited as a soldier".
} 
Secretario General a quien se encomiendan una serie de misiones a ese objeto, las que se han ido ejecutando en el tiempo ${ }^{17}$.

Ahora bien, lo establecido en la Resolución 1325 ha sido complementado y expandido por medio de otras Resoluciones del Consejo de Seguridad: la 1820, del año 2008; las 1888 y 1889 , ambas de 2009; la 1960, del año 2010; las 2106 y 2122, ambas del año 2013; y la Resolución 2242, del año $2015^{18}$.

La Resolución 1820 condena el uso de la violación y otras formas de violencia sexual en situaciones de conflicto, declarando que la violación puede constituir un crimen de guerra, un crimen contra la humanidad o un acto constitutivo de un genocidio, y pide a los estados miembros enjuiciar a los autores de violencia sexual, para garantizar que todas las víctimas de esta violencia tengan igual protección ante la ley e igual acceso a la justicia ${ }^{19}$, siendo coherente con el desarrollo del tema en el marco del derecho internacional de los derechos humanos ${ }^{20}$.

La Resolución 1888, por su lado, complementa la 1820 y pide al Secretario General desplegar un equipo de expertos en el tema y que se nombre un representante especial para dirigir los esfuerzos para poner fin a la violencia sexual contra mujeres y niños en conflicto y se ocupe de situaciones particularmente preocupantes, colaborando con la presencia de la ONU sobre el terreno y previo consentimiento del gobierno receptor, a fin de ayudar a las autoridades nacionales a reforzar el estado de derecho, lo cual se desarrolló efectivamente en el año $2010^{21}$. La Resolución 1889, por su parte, reafirma la 1325, instando a los estados a su implementación efectiva, y llama al Secretario General de Naciones Unidas a desarrollar una estrategia que incluya un adecuado entrenamiento, para aumentar el número de mujeres para ejercer buenos oficios en su nombre y para que en el plazo de seis meses presentara indicadores de

17 Así las cosas, y en conformidad con la Resolución 1325, el Secretario General de la ONU presentó en 2002 un estudio llamado "Mujeres, Paz y Seguridad" que vino a establecer un verdadero marco teórico por el que los países y en general toda la institucionalidad internacional que trabaja en torno al tema debe guiarse en la materia. Es interesante destacar cómo este documento se hace cargo sin quererlo del problema clásico de confusión entre "estudios de género" y "estudios sobre mujeres", usando una fórmula que libera de responsabilidad en cuanto a que sólo considera el tema en clave de mujer. Dice el párrafo 50 “...there is often confusion and misunderstanding of whether or not a gender analysis is the same as a focus on women. This study focuses on the experiences of women and girls. It has, however, used gender analysis as the basis for understanding what happens to women and girls in armed conflict and to develop effective operational responses". Women, Peace and Security, Study submitted by the Secretary-General pursuant to Security Council Resolution 1325 (2000) United Nations, 2002. http://www.un.org/womenwatch/daw/public/eWPS.pdf, (consultado el 20 de junio de 2016).

18 Ello sin perjuicio de las referencias que muchas resoluciones del citado Consejo hacen periódicamente a la situación de las mujeres y la paz, a propósito de determinados asuntos. Así por ejemplo, en 2014, 38 de las 63 resoluciones aprobadas por el CSNU tenían esta referencia, lo que equivale a un 60,3\%. Informe S/2015/716 (2015), párrafo 148.

19 Resolución S/RES/1820 del Consejo de Seguridad de las Naciones Unidas, 19 de junio de 2008.

20 En efecto, el Comité para la Eliminación de toda forma de discriminación contra la mujer de Naciones Unidas ha señalado que con base en los artículos 2, 5, 11, 12 y 16 de la Convención para la eliminación de toda forma de discriminación contra la mujer los estados partes están obligados a proteger a las mujeres contra cualquier tipo de violencia, en cualquier ámbito de la vida social en que ésta se produzca. Agrega además que la violencia contra la mujer es una forma de discriminación que impide gravemente el goce de derechos y libertades en pie de igualdad con el hombre. Comité para la Eliminación de toda forma de discriminación contra la mujer. Recomendación General Nº 12, de 1989 y Recomendación General N 19 , de 1992.

21 Resolución S/RES/1888 del Consejo de Seguridad de las Naciones Unidas, 30 de septiembre de 2009. 
La Resolución 1325 del Consejo de Seguridad de las Naciones Unidas. A 15 años de su adopción

Ximena Gauché Marchetti

\author{
seguimiento de implementación de la Resolución $1325^{22}$, indicadores sobre los cuales los \\ estados han ido basando sus estrategias de implementación ${ }^{23}$.
}

22 Resolución S/RES/1889 del Consejo de Seguridad de las Naciones Unidas, 5 de octubre de 2009.

23 Informe S/2010/173 del Secretario General de las Naciones Unidas. Ese Informe de indicadores fue presentado en abril de 2010 dando cuenta que todas las actividades de las entidades de las Naciones Unidas en el marco del Plan de acción de 2008-2009 (Informe S/2007/567) para todo el sistema ONU se organizaron en torno a cinco ámbitos temáticos: prevención; participación; protección; socorro y recuperación; y aspectos normativos, estableciéndose respecto de cada una de las cuatro primeras cierto número de indicadores de vigilancia y entendiendo que el último es de carácter intersectorial y quedaría incorporado en los otros cuatro. Este trabajo fue el resultado de un proceso amplio e inclusivo para reunir información sobre los indicadores que se estaban utilizando en todo el sistema de las Naciones Unidas y que empleaban los gobiernos nacionales y otras organizaciones. Cada uno de los indicadores propuestos está vinculado a un objetivo concreto. En el pilar de Prevención los indicadores son: Indicador 1: Incidencia de la violencia sexual en los países afectados por conflictos; Indicador 2: Medida en que las misiones de mantenimiento de la paz y misiones políticas especiales de las Naciones Unidas incluyen en sus informes periódicos información sobre las violaciones de los derechos humanos de las mujeres y niñas; Indicador 3 a): Número de violaciones de los derechos humanos de las mujeres y niñas que son denunciadas, remitidas a los órganos de derechos humanos e investigadas por éstos Indicador 3 b): Inclusión de representantes de las organizaciones de mujeres y de la sociedad civil en el personal directivo y de gestión de los órganos de derechos humanos; Indicador 4: Porcentaje de casos denunciados de explotación y abuso sexual presuntamente cometidos por personal uniformado y civil encargado del mantenimiento de la paz y trabajadores humanitarios, que son remitidos a la justicia e investigados y sobre los que se adoptan medidas concretas; Indicador 5 a): Número y porcentaje de directrices para el personal de mantenimiento de la paz emitidas por los jefes de los componentes militares y de procedimientos operativos estándar que comprenden medidas para proteger los derechos humanos de las mujeres y niñas y 5 b): Número y porcentaje de manuales militares, marcos normativos de seguridad nacional, códigos de conducta y procedimientos operativos estándar o protocolos de las fuerzas de seguridad nacionales que comprenden medidas para proteger los derechos humanos de las mujeres y niñas; Indicador 6: Número y tipo de medidas adoptadas por el Consejo de Seguridad en relación con la resolución 1325 (2000), incluidas las medidas encaminadas a prevenir y castigar las violaciones de los derechos humanos de las mujeres y niñas en situaciones de conflicto; e Indicador 7: Número y proporción de mujeres que ocupan cargos directivos en las organizaciones regionales pertinentes que se ocupan de la prevención de conflictos. Los indicadores correspondientes al pilar de la participación son: Indicador 8: Número y porcentaje de los acuerdos de paz que contienen disposiciones concretas para mejorar la seguridad y la condición de las mujeres y niñas; Indicador 9: Número y porcentaje de mujeres que ocupan altos cargos directivos en las Naciones Unidas en los países afectados por conflictos; Indicador 10: Grado de especialización en cuestiones de género en la toma de decisiones de las Naciones Unidas en los países afectados por conflictos; Indicador 11 a): Nivel de participación de las mujeres en las negociaciones de paz oficiales y $11 \mathrm{~b}$ ): Presencia de las mujeres en cargos de observadoras oficiales o de carácter consultivo al comienzo y al final de las negociaciones de paz; Indicador 12: Nivel de participación política de las mujeres en los países afectados por conflictos; Indicador 13: Número y porcentaje de misiones del Consejo de Seguridad que tienen presentes en su mandato y en sus informes los problemas concretos que afectan a las mujeres y las niñas. El tercer pilar es la protección y en él se encuentran como indicadores: Indicador 14: Índice de seguridad física de las mujeres y niñas; Indicador 15: Medida en que las leyes nacionales protegen los derechos humanos de las mujeres y las niñas, de conformidad con las normas internacionales; Indicador 16: Nivel de participación de las mujeres en el sector de la justicia y la seguridad en los países afectados por conflictos; Indicador 17: Existencia de mecanismos nacionales de control de las armas pequeñas y armas ligeras; Indicador 18: Porcentaje de mujeres en la población adulta empleada en los programas para una pronta recuperación económica; Indicador 19: Número y porcentaje de casos de violencia sexual contra las mujeres y niñas que son remitidos a la justicia e investigados y sobre los que se pronuncia sentencia; e Indicador 20: Número y porcentaje de tribunales dotados para conocer casos de violaciones de los derechos humanos de las mujeres y niñas, con la debida atención a la seguridad de las víctimas. En el pilar del socorro y recuperación se observan los siguientes: Indicador 21 a): Mortalidad materna y 21 b): Tasas de matrícula en la enseñanza primaria y secundaria, desglosadas por sexo; Indicador 22: Medida en que se incorporan en los marcos de planificación estratégicos de los países afectados por conflictos análisis, objetivos, indicadores y presupuestos relacionados con el género; Indicador 23: Proporción de la financiación asignada y desembolsada por las organizaciones de la sociedad civil, incluidos los grupos de mujeres, que se dedica a las cuestiones de género en los países afectados por conflictos; Indicador 24: Monto efectivo de la financiación asignada y desembolsada para apoyar programas de socorro, recuperación, paz y seguridad en que se tengan en cuenta las cuestiones de género, en los países afectados por conflictos; Indicador 25 a): Número y porcentaje de mecanismos de la justicia de transición establecidos en los procesos de paz que comprenden en sus mandatos disposiciones para proteger los derechos y fomentar la participación de las mujeres y niñas y $25 \mathrm{~b}$ ): Número y porcentaje de mujeres y niñas que se benefician de los programas de resarcimiento y tipos de beneficios recibidos; e Indicador 26: Número y porcentaje de mujeres excombatientes y de mujeres y niñas asociadas con las fuerzas o los grupos armados que se benefician de los programas de desarme, desmovilización y reintegración. Véase el detalle y desarrollo de tales indicadores en S/2010/173, párrafos 12-43. La lista de indicadores que figura en este informe representa la culminación de 
En el año 2010, en la Resolución 1960 se reafirma que la violencia sexual, cuando se utiliza o se hace utilizar como táctica de guerra o como parte de un ataque generalizado o sistemático contra la población civil, puede prolongar y agudizar significativamente las situaciones de conflicto armado y constituir en algunos casos un impedimento para el restablecimiento de la paz y la seguridad internacionales. Afirma, a este respecto, que la adopción de medidas eficaces para prevenir los actos de violencia sexual y responder a ellos puede contribuir considerablemente al mantenimiento de la paz y la seguridad internacionales, y manifiesta que está dispuesto a adoptar, cuando considere situaciones sometidas a su examen, según sea necesario, medidas apropiadas para hacer frente a la violencia sexual generalizada o sistemática en situaciones de conflicto armado, estableciendo luego la solicitud al Secretario General para seguir trabajando en el tema, que queda entonces constituido como preferente ${ }^{24}$.

En 2013, la Resolución 2106 del Consejo de Seguridad reitera que todos los Estados miembros y las entidades de las Naciones Unidas deben hacer más para implementar los mandatos anteriores y combatir la impunidad de la violencia sexual relacionada con el conflicto ${ }^{25}$. Por su parte, en la Resolución 2122 el órgano encargado de la seguridad internacional puso énfasis en la rendición de cuentas sobre la aplicación de la Resolución 1325, y la importancia de la participación de las mujeres en todas las fases de la prevención de conflictos, resolución y recuperación ${ }^{26}$.

En el año 2015, con ocasión de la conmemoración de los 15 años de la Resolución 1325 y del Informe del Secretario General sobre resultados de su implementación, de los 20 años de la Conferencia de Beiging, de la adopción de los Objetivos de Desarrollo Sostenible y coincidiendo con el 70 aniversario de las Naciones Unidas, se adoptó la Resolución $2242^{27}$. Ella marca el inicio de una nueva etapa que profundiza recomendaciones y exhortaciones a los estados, a partir de lo logrado y no logrado en los primeros quince años de vida de la Resolución 1325.

En el apartado siguiente se hará una revisión de algunos resultados de la implementación de tal Resolución 1325 y las posteriores, teniendo en vista el discurso oficial de diversos órganos de las propias Naciones Unidas, así como otras fuentes que permitan mostrar la evaluación desde el 2000 a la fecha.

un proceso de consulta extenso, con una amplia participación y abrió espacio para que desde 2010 y en el marco de los diez años de la Resolución 1325, los organismos de la familia de NU y los países trabajaran a partir de ellos para su plena implementación, lo que se tuvo en vista en el curso del año 2015, de cara a la conmemoración de los 15 años de la Resolución 1325. Se observa en ellos en todo caso la ausencia de algunos temas relevantes en el contexto de la seguridad internacional. Ejemplos son el tema de las mujeres como refugiadas y desplazadas; la cuestión de su consideración en los procesos de justicia transicional; o su participación en procesos políticos propios de las gobernanzas locales, cuestiones que quizás pueden entenderse comprendidas en algunos de los indicadores citados pero que por su relevancia habrían ameritado tal vez una mención específica.

24 Resolución S/RES/1960 del Consejo de Seguridad de las Naciones Unidas, 16 de diciembre de 2010.

25 Resolución S/RES/2106 del Consejo de Seguridad de las Naciones Unidas, 24 de junio de 2013.

26 Resolución S/RES/2122 del Consejo de Seguridad de las Naciones Unidas, 18 de octubre de 2013.

27 Resolución S/RES/2242 del Consejo de Seguridad de las Naciones Unidas, 13 de octubre de 2015. 
La Resolución 1325 del Consejo de Seguridad de las Naciones Unidas. A 15 años de su adopción Ximena Gauché Marchetti

\section{A 15 años de la Resolución 1325: la evaluación institucional de sus resultados.}

Lograr la igualdad de las mujeres es hoy tema de la agenda formal del Consejo de Seguridad de las Naciones Unidas. Desde ya podría decirse que ese es el gran logro que exhibe la Resolución 1325 del citado órgano, fruto de lo cual existen hoy 54 planes de acción nacional y una serie de estrategias formales $^{28}$, no obstante las críticas que el lenguaje o las estrategias de ONU ha producido en muchos casos con interesantes fundamentos desde la teoría del género ${ }^{29}$. Además, este discurso ha llegado con fuerza al trabajo del Secretario General a quien además se ha sumado activamente ONU Mujeres desde su creación en el año $2010^{30}$.

Sin embargo, es frecuente que Naciones Unidas en sus 70 años de vida haya adoptado temas en la agenda institucional sin lograr resultados efectivos.

Los Objetivos de Desarrollo del Milenio (ODM) son un buen ejemplo de ello. Las 8 metas para 2015, adoptadas en el año 2000 con ocasión de la Cumbre del Milenio, buscaban esencialmente liberar a la humanidad de la pobreza extrema (en sus múltiples dimensiones) y erradicar otros males: la falta de enseñanza primaria universal, la desigualdad entre los géneros y la falta de empoderamiento de la mujer, la mortalidad infantil en menores de 5 años, los problemas en salud materna, el VIH/Sida, el paludismo y otras enfermedades, y lograr la sostenibilidad del medio ambiente, fomentando además una alianza mundial para el desarrollo. A pesar de los loables esfuerzos y las múltiples estrategias globales, nacionales y locales por cumplir las metas e indicadores planteados para cada objetivo, al año 2015 persisten notables

28 Una de las maneras que tienen los gobiernos nacionales de aplicar las resoluciones sobre las mujeres, la paz y la seguridad es mediante la elaboración de Planes de Acción Nacional (en adelante, PAN). De esta forma, un PAN se configura como un documento que detalla las medidas que está adoptando actualmente un gobierno, y las iniciativas que llevará a cabo en un plazo de tiempo determinado para cumplir las obligaciones incluidas en la Resolución 1325. Se trata de la iniciativa que guarda una mayor orgánica desde el punto de vista formal para la implementación de los estándares internacionales que se han ido fijando en la materia en estos quince años, debiendo tener sus estrategias un carácter integrado. No obstante, además de los PAN sobre las mujeres, la paz y la seguridad, existen otros medios o estrategias particulares para implementar tales resoluciones y estándares, como son por ejemplo, integrar los elementos clave de las resoluciones sobre las mujeres, la paz y la seguridad en los planes y políticas de los ministerios nacionales relacionados, tales como, Defensa, Interior, Justicia, Economía y Desarrollo Social, Género, Desarrollo y Relaciones Exteriores o, partir del proceso de elaboración de un PAN, impulsar asignaciones presupuestarias y medidas en el seno de cada uno de los ministerios y reparticiones pertinentes con el propósito de promover los objetivos de las mujeres, la paz y la seguridad, o trabajar directamente en la integración de mujeres en las operaciones de paz en que participan los países, en el marco a su vez de los cambios que estas operaciones siguen experimentando a nivel mundial. Chile ostenta el honor de haber sido el primer país del continente en elaborar e implementar un PAN durante la vigencia del primer gobierno de Michelle Bachelet y también el primero del continente en dotarse de un segundo plan, previsto para ser implementado entre 2015 y 2018.

29 Por ejemplo, el uso de género ha sido criticado en el contexto de las operaciones de paz como algo solo asociado a mujeres y con una irrelevancia del tema para los hombres pues se centraría en lograr una mayor participación de mujeres y no en cambiar espacios y discursos masculinizados como son aquellos que tienen que ver con seguridad. Véase sobre este punto en Solhjell Randi, “'Sin mujeres por aquí. Discursos de género en las operaciones de paz de Naciones Unidas”, Revista Relaciones Internacionales 27 (octubre 2014-enero 2015): 97-116.

30 ONU MUJERES fue creada por medio de la Resolución 64/289 de la Asamblea General. 
focos de desigualdad y si bien hay cifras auspiciosas que se pueden mostrar, todavía se mantienen la mayoría de los males que se buscaba erradicar y han surgido otros, como es el caso de los desplazamientos humanos forzados. En efecto, según el informe final oficial de cumplimiento de los ODM elaborado por Naciones Unidas en 2015 se estima a la fecha que 836 millones de personas viven todavía en pobreza extrema; 6 millones de niños menores de 5 años siguen muriendo; todavía casi un $10 \%$ de la población mundial no usa una fuente de agua mejorada; un 29,7\% de población urbana vive en barrios marginales en regiones en desarrollo y solo 1 de cada 5 miembros de un parlamento nacional es mujer ${ }^{31}$.

Así las cosas, bien vale preguntarse entonces si la inclusión del tema de las mujeres en la agenda de paz y seguridad desde el año 2000 ha tenido logros efectivos que puedan ser relevados, más allá de la adopción de planes de acción nacional u otras estrategias del nivel global, regional o nacional.

La pregunta anterior tiene especial relevancia de cara a lo que fue el año 2015 para las Naciones Unidas. Un año en que la organización cumplió 70 años, se conmemoraron 15 años de la Resolución 1325, se evaluaron los resultados de los ODM y se proyectó una agenda mundial sobre desarrollo sostenible de cara al año 2030. Tal como ocurrió al planificarse la agenda del Milenio, la del desarrollo sostenible puso el tema de la igualdad de género como uno relevante. Ello se desprende al constatar que de los 17 objetivos, el quinto sea la igualdad de género y el empoderamiento de la mujer, planteando una serie de metas que tienen directa relación con lo que se propuso en 2000 el Consejo de Seguridad al adoptar la Resolución $1325^{32}$.

Por esto, resulta útil establecer qué evaluación global se hizo en 2015 desde las propias Naciones Unidas. Para ello se ofrece en este trabajo una descripción genérica a partir de las cuatro categorías generales de mandatos que se engloban en las resoluciones del CSNU como ha puesto en evidencia ONU Mujeres ${ }^{33}$ : la participación, la prevención, la protección y la consolidación de la paz. La participación tiene que ver con la inclusión de mujeres en los procesos de paz y todos los procesos de toma de decisiones pública relacionados con establecer y consolidar la paz. La prevención a su vez con la incorporación de las perspectivas de las mujeres en sistemas de alerta temprana, educación pública, y el enjuiciamiento de aquellas personas que violen los derechos de las mujeres. La protección de las mujeres tiene que ver con su resguardo durante el conflicto y después del mismo por parte de personal de seguridad comunitario, nacional e internacional. Finalmente, la consolidación de la paz implica que se tengan en cuenta a las mujeres y sus necesidades en las actividades de socorro y

31 Naciones Unidas, Objetivos de Desarrollo del Milenio, 2015, 4-7, http://www.un.org/es/millenniumgoals/pdf/2015/mdg-report-2015_spanish.pdf (consultado el 20 junio de 2016).

32 Naciones Unidas, "Objetivos del Desarrollo sostenible: Objetivo 5: Lograr la igualdad entre los géneros y empoderar a todas las mujeres y las niñas", http://www.un.org/sustainabledevelopment/es/gender-equality/ (consultado el 20 de junio de 2016).

33 ONU MUJERES, Las mujeres, la paz y la seguridad: directrices para la implementación nacional, 2012, p.6. 
La Resolución 1325 del Consejo de Seguridad de las Naciones Unidas. A 15 años de su adopción Ximena Gauché Marchetti

recuperación, resarcimiento por injusticias sufridas e inversión en seguridad social y económica.

En materia de participación, se puede atribuir como mérito de la Resolución 1325 haber instalado de forma definitiva la cuestión de la inclusión de mujeres y asuntos de género en los trabajos formales para la construcción, el mantenimiento y la consolidación de la paz. Ello ha significado en la práctica, por ejemplo, que algunas mujeres hayan desempeñado cargos influyentes por encargo del Secretario General en algunas misiones de paz, como es el caso de la embajadora danesa Ellen Mergrethe Loj, quien actuó como su Representante Especial para Liberia $^{34}$; Ameerah Haq, Jefa Adjunta, Coordinadora Humanitaria y Coordinadora Residente de la Misión en Sudán y su Representante Especial para Timor Leste en su tiempo ${ }^{35}$; o Bintou Keita, quien ejerció de Representante Ejecutiva del Secretario General en Burundi ${ }^{36}$. También, la incorporación de mujeres a las operaciones de paz de Naciones Unidas, en que se ha pasado de 0 en el año 2000 a 3.332 integrantes mujeres al año 2010 (un pequeño 3,33\%) ) $^{37}$ y con una consolidada incorporación del género en todas las actuales operaciones multidimensionales de paz $^{38}$. Claro eso sí, que mayoritariamente empleadas como personal de apoyo ${ }^{39}$. Además, con ocasión de los debates que se dieron en el segundo semestre de 2015 para examinar la agenda del Consejo de Seguridad en materia de mujeres, paz y seguridad y conmemorar los 15 años de la Resolución 1325, ONU Mujeres daba cuenta de que para 2014 el 88\% de los procesos de paz en que participó la organización incluyó consulta a mujeres, lo que representa un aumento del $50 \%$ en relación a 2011 y una cifra muy superior al 11\% que refería a mujeres o género antes del año 2000. Sin embargo, la cifra global muestra que al 2015, sólo un cuarto del total de acuerdos de paz tenía referencia a mujeres ${ }^{40}$. Ello no obstante que algunas investigaciones han dado cuenta de la correlación positiva entre participación efectiva y significativa de las mujeres en los procesos, por un lado, y sostenibilidad de las conversaciones y la aplicación del acuerdo, por el otro ${ }^{41}$. Además, no siempre el aumento estadístico de participación va acompañado de influencia femenina en los procesos de negociación u otros aspectos ${ }^{42}$.

Esto último parece consecuente con que en materia de toma de decisiones de alta política en puestos de liderazgo aún permanecen las mujeres infra representadas. A 2015, sólo el 16\% de

4 SG/A/1206 BIO/4148, 2 de diciembre de 2009.

35 SG/A1104 AFR/1603 BIO/3946, 24 de octubre de 2007.

36 ONU MUJERES, Las mujeres, la paz y la seguridad: directrices para la implementación nacional, 2012.

37 Mantenimiento de la paz de las Naciones Unidas, "Estadísticas de mantenimiento de la paz", Naciones Unidas, http://www.un.org/es/peacekeeping/resources/statistics/ (consultado el 20 de junio de 2016).

38 S/2015/716 (2015), párrafo 77.

39 S/2015/716 (2015), párrafo 80.

40 ONU MUJERES, "Comunicado de prensa: En un clima de cada vez más extremismo, el papel de las mujeres en la paz y la recuperación todavía se enfrenta a obstáculos y está infrautilizado", Naciones Unidas,

http://www.unwomen.org/es/news/stories/2015/10/press-release-womens-role-in-peace-and-recovery (consultado el 20 de junio de 2016).

41 S/2015/716 (2015), párrafo 11.

42 Radhika Coomaraswamy, “A Global Study on the Implementation of United Nations Security Council resolution 1325”, Preventing conflict, transforming justice, securing the peace (2015), 45, http://wps.unwomen.org/ /media/files/un\%20women/wps/highlights/unw-global-study-1325-2015.pdf (consultado el 20 de junio de 2016). 
los presidentes de Parlamentos nacionales son mujeres y representan sólo el 18\% de todos los ministerios del mundo ${ }^{43}$. El tema no es menor si pensamos que es en los parlamentos nacionales y luego desde algunos ministerios estratégicos desde donde deben surgir las normas y políticas, planes o programas que las implementen. Naciones Unidas está consciente de ello y prueba es que decidió conmemorar los ODM usando el lema "Empoderando a las mujeres. Empoderando a la humanidad. ¡Imagínalo!” con el que se busca recrear un mundo en que cada mujer y cada niña pueda elegir sus decisiones, sea en el ámbito de la política o la educación, y tener sus propias vivencias en sociedades libres de discriminación y de violencia ${ }^{44}$. El Secretario General, a su vez, ha dado cuenta de la relación inversamente proporcional entre el número de parlamentarias y los niveles de corrupción, y que los órganos de decisión inclusivos abordan una mayor cantidad de problemas, incluyendo derechos y servicios esenciales básicos como educación, salud o reformas con enfoque de género ${ }^{45}$.

En el ámbito de la prevención, investigaciones recientes indican que los niveles de igualdad de género y acceso de la mujer a los derechos económicos, políticos y sociales, así como de la seguridad de las mujeres, son indicadores clave del grado de paz de un Estado. Por tanto, una mayor inversión en la igualdad entre los géneros y el empoderamiento de la mujer, en particular en lo que respecta a las oportunidades educativas y de capacitación, están intrínsecamente vinculados a la estabilidad, el desarrollo, la paz y los derechos humanos ${ }^{46}$. Por otra parte, siempre en el ámbito de la prevención, un logro interesante es la entrada en vigor del Estatuto de Roma que crea la Corte Penal Internacional, tribunal permanente que tiene en cuenta el género en el juzgamiento de los crímenes que tipifica, dando por vez primera en un tratado de esa naturaleza una definición de género ${ }^{47}$ y tipificando algunas conductas. Además,

43 Naciones Unidas, Objetivos de Desarrollo del Milenio, 2015, 31.

http://www.un.org/es/millenniumgoals/pdf/2015/mdg-report-2015_spanish.pdf (consultado el 20 de junio de 2016).

44 Naciones Unidas, "Día Internacional de la Mujer 8 de marzo", http://www.un.org/es/events/womensday/ (consultado el 20 de junio de 2016).

45 S/2015/716, (2015), párrafo 28.

$46 \quad \mathrm{~S} / 2015 / 716$ (2015), párrafo 67.

47 La definición de género está contenida en el artículo 7.3 en el marco de la regulación de los crímenes de lesa humanidad e indica que "A los efectos del presente Estatuto se entenderá que el término género se refiere a los dos sexos, masculino y femenino, en el contexto de la sociedad. El término género no tendrá más acepción que la que antecede". Fuera de este artículo, el Estatuto usa la expresión género en varias otras disposiciones: en materia del derecho aplicable (artículo 21.3); a propósito de la regulación de la Fiscalía (artículo 9); en las funciones y atribuciones del Fiscal con respecto a las investigaciones (artículo 54, 1 letra b) y en lo que toca a la protección de las víctimas y los testigos y su participación en las actuaciones (artículo 68). La definición de género del Estatuto de Roma ha sido objeto de fundadas críticas por la doctrina internacionalista preocupada de los temas de mujeres y género ya que, siendo justos con sus méritos, la verdad es que no dejó de lado las ambigüedades que la idea de género y su relación con sexo han tenido y tienen a la hora de ser abordadas por Naciones Unidas, sino que por el contrario parece haberlas agudizado, constituyendo en verdad lo que la doctrina llama el uso de la "ambigüedad constructiva" por parte de los negociadores, entendiendo que este término se utiliza en diplomacia para referir al uso de palabras ambiguas que dan consuelo a las diferentes partes que intervienen en el debate, promoviendo así el acuerdo. A partir de la investigación en que se enmarca este artículo puede indicarse que estas críticas se reconducen a las siguientes: la fusión de las ideas de género y sexo, colapsando la primera a favor de una concepción biológica y estática; y la exclusión en la forma en que se concibe el género, de otras identidades de género y además de las diversas orientaciones sexuales. Sobre la primera de estas cuestiones, como lo señala una participante del proceso de redacción y adopción del concepto de género en el Estatuto, la cuestión es si el uso de las expresiones "los dos sexos, masculino y femenino" hace colapsar la idea de género hacia la idea de sexo, lo cual no es nada menor pues indicará qué factores tendrá que tener en cuenta la Corte Penal al examinar los crímenes que juzgue y las necesidades de los testigos, por ejemplo. Al parecer, la lectura descontextualizada podría dar a entender que sólo deben ser considerado el hombre y la mujer como los dos sexos que 
La Resolución 1325 del Consejo de Seguridad de las Naciones Unidas. A 15 años de su adopción

Ximena Gauché Marchetti

se incluye la obligación de tener en cuenta en la selección de los magistrados internacionales una "representación equilibrada de magistrados hombres y mujeres" como dice el artículo 36.8.a).III. Este modelo complementa los nacionales y fortalece el trabajo de las comisiones de verdad ${ }^{48}$.

En el área de la protección de derechos de las mujeres y niñas durante el conflicto y después del mismo, el año 2015 ha demostrado que el discurso normativo se encuentra disociado de la realidad y que hay pocos avances que reportar. En efecto, ha quedado en evidencia que en situaciones de crisis y emergencia ellas tienen menos posibilidades de disfrutar derechos como la alimentación, salud, educación, vivienda, e incluso nacionalidad ${ }^{49}$. Además, que en materia de derecho a la vida y a la integridad física no hay logros pues la violencia en diversas formas es una constante hoy en los conflictos, desprotección que se agrava en situaciones de desplazamiento y refugio por la mayor indefensión a que están expuestas las mujeres ${ }^{50}$.

Por cierto, el triste fenómeno de la violencia hacia mujeres es uno creciente no sólo en los contextos de conflicto. Ello ha motivado el trabajo desde diferentes frentes institucionales para prevenirla, erradicarla y sancionarla. La Comisión Económica para América Latina y el Caribe (CEPAL) indica que las Naciones Unidas identifica y tipifica una amplia variedad de formas de violencia contra las mujeres, con diferentes escenarios, ámbitos y caracterización. En ese sentido los principales escenarios son la violencia dentro de la familia, en la comunidad, cometida o consentida por el Estado, en conflictos armados y en el marco de la discriminación múltiple que puede afectarles. En el caso específico de la violencia que se produce en conflictos armados ella se caracteriza por hechos como los homicidios, torturas y otros tratos crueles, inhumanos o degradantes, raptos, mutilaciones y desfiguraciones, reclutamiento forzado de mujeres combatientes, violencia sexual, violaciones, esclavitud sexual, explotación

existen, no teniendo género ningún significado que vaya más allá de lo biológico y por tanto no cabría entender que, por ejemplo, se incluya a los transgéneros o a los intersexuales. Sin embargo, la lectura contextualizada justamente con la expresión "en el contexto de la sociedad" ha de llevar a que entonces lo biológico es un punto de partida pero no se configura como un determinismo con extremos en los que necesariamente haya que moverse. Frente a ello, parece que las opciones de la Corte Penal tendrían que girar entre estas indicadas, pronunciándome junto a una autora en el sentido favorable a la segunda por las dos razones que se esgrimen. En primer lugar, la historia de la elaboración del Estatuto muestra que no se pudo dejar establecido con acuerdo esta especie de determinismo biológico querido por algunos; y en segundo lugar, considerando el contexto que brinda el derecho internacional hay que tomar en cuenta la incorporación de la perspectiva de género en este trabajo. Darle un interpretación así amplia a mi entender también resulta coherente con las disposiciones del régimen de Viena sobre interpretación de tratados y bajo la aceptación de la regla del efecto útil que debería darse a toda norma. Véase Valerie Oosterveld, "The Definition of "Gender" in the Rome Statute of the International Criminal Court: A step forward or back for International Criminal Justice”, Harvard Human Rights Journal 18 (2005). Una reseña sobre el proceso de negociación del Estatuto de Roma y la historia para incluir la expresión género véase específicamente en pp.58-66.

48 En los últimos 15 años, las comisiones de verdad de Perú (2001-2003), Timor-Leste (2001-2006) y Sierra Leona (20022004) han sido pioneras en integrar una perspectiva de género en sus trabajos. Véase Radhika Coomaraswamy, "A Global Study on the Implementation of United Nations Security Council resolution 1325", Preventing conflict, transforming justice, securing the peace (2015), 111, http://wps.unwomen.org/ /media/files/un\%20women/wps/highlights/unw-global-study1325-2015.pdf (consultado el 20 de junio de 2016).

49 Radhika Coomaraswamy, "A Global Study on the Implementation of United Nations Security Council resolution 1325”, Preventing conflict, transforming justice, securing the peace (2015), 74-88, http://wps.unwomen.org/ /media/files/un\%20women/wps/highlights/unw-global-study-1325-2015.pdf (consultado el 20 de junio de 2016).

50 S/2015/716 (2015), párrafos 39 al 48. 
sexual, desapariciones forzadas, prisiones arbitrarias, matrimonios forzados, prostitución forzada, abortos forzados, embarazos forzados y esterilización compulsiva ${ }^{51}$.

Finalmente, en el ámbito de la consolidación de la paz el gran avance es la consideración clara de que una paz y seguridad verdadera sólo se consiguen incorporando a las mujeres en los procesos de reconstrucción una vez que ha concluido un conflicto, elaborando estrategias para ese fin.

En esa línea, en 2010 el Secretario General propuso a la Asamblea General y al Consejo de Seguridad de la ONU un Plan de Acción para la consolidación de la paz con perspectiva de género $^{52}$, en que se establecieron siete compromisos con medidas y actividades de apoyo específicas con carácter colaborativo entre todos los actores involucrados. Estos compromisos se referían a la participación de la mujer en solución de los conflictos ${ }^{53}$, a la planificación de la colaboración en los procesos de transición ${ }^{54}$, la financiación ${ }^{55}$, el despliegue de capacidad civil ${ }^{56}$, a los métodos destinados a aumentar la proporción de mujeres encargadas de la adopción de decisiones en las instituciones de gobernanza después de los conflictos ${ }^{57}$, apoyo al estado de derecho y a las instituciones que prestan seguridad, justicia y legislan ${ }^{58} \mathrm{y}$, por último, sobre recuperación económica ${ }^{59}$.

Para 2015, el propio Secretario General tuvo que reconocer que varias de las medidas establecidas en el plan no se han aplicado plenamente, limitando así las posibilidades de las mujeres de participar en procesos de consolidación de la paz ${ }^{60}$. Específicamente en tres áreas reportó los mayores atrasos. En primer lugar, en la recuperación económica y el acceso a los recursos, en que se ha constatado que tales recursos y la inversión extranjera siguen llegando a espacios en que las mujeres están sub-representadas como son, por ejemplo, la infraestructura o las industrias extractivas, o que el empoderamiento de las mujeres no alcanza a llevarse el 5\% del gasto en recuperación económica, lo que contribuye a mantenerlas en el microcrédito, la economía informal o como receptoras de empleos temporales ${ }^{61}$. En segundo término, en materia de gobernanza y participación en órganos electivos y no electivos, en que se constató que existe aún una menor participación decisoria en los países que salen de conflicto en los cargos de elección pero que existiendo igual infra representación en cargos designados o cargos en la administración pública, no se ha tomado la misma conciencia sobre ello, existiendo

\footnotetext{
51 Comisión Económica para América Latina y el Caribe (CEPAL), Informe anual 2013-2014, El enfrentamiento de la violencia contra las mujeres en América Latina y el Caribe (2014), 27.

52 Informe A/65/354-S/2010/466 del Secretario General de las Naciones Unidas, 7 de septiembre de 2010.

A/65/354-S/2010/466 (2010), párrafos 27 y 28.

A/65/354-S/2010/466 (2010), párrafos 29 al 33.

A/65/354-S/2010/466 (2010), párrafos 34 al 36.

A/65/354-S/2010/466 (2010), párrafos 37 al 39.

A/65/354-S/2010/466 (2010), párrafos 40 al 44.

A/65/354-S/2010/466 (2010), párrafos 45 al 48

A/65/354-S/2010/466 (2010), párrafos 49 al 52.

S/2015/716 (2015), párrafo 23.

S/2015/716 (2015), párrafos 25 a 27.
} 
La Resolución 1325 del Consejo de Seguridad de las Naciones Unidas. A 15 años de su adopción Ximena Gauché Marchetti

además falta de datos sobre la cantidad de ellas actuando en los procesos de reconstrucción ${ }^{62}$. Finalmente, el sector desarme, desmovilización y reintegración, y la reforma a la seguridad es un ámbito de la consolidación de la paz en que no se han logrado avances sustantivos, sino más bien menores. Por ejemplo, en países que han salido de conflictos el presupuesto para proyectos de igualdad de género en el marco de las reformas a la seguridad fue de menos del $1 \%$, sumado ello a una insuficiente rendición de cuentas; la cifra de mujeres participando en programas de desarme, desmovilización y reintegración ha aumentado pero no hay indicadores sobre calidad o efectos en la vida de las mujeres ${ }^{63}$.

\section{A modo de cierre, ideas finales sobre el futuro de la Resolución 1325 y sucesoras en el escenario internacional actual.}

Usando aquel conocido refrán que invita a mirar el vaso medio lleno y no medio vacío, la primera reflexión es positiva. La Resolución 1325 y sus sucesoras han significado un cambio notable en el discurso oficial de la seguridad y la paz internacional y ello ha permeado el trabajo de organismos internacionales y de muchos países.

Sin embargo, como ha reconocido el Secretario General de la $\mathrm{ONU}^{64}$, para 2015 el mundo tiene un panorama desolador en materia de paz y seguridad, con violaciones flagrantes a derechos humanos y al derecho humanitario, con factores complejos como desencadenantes de los conflictos y la intervención en ellos de un número cada vez mayor de agentes no estatales, nuevas tecnologías y conexiones transnacionales que, evidentemente tienen una incidencia en la naturaleza de los conflictos y afecta a las mujeres de forma específica.

En ese sentido, el vaso está medio vacío y coincide con el desolador panorama mundial del último período que muestra el Informe Anual 2015/2016 de Amnistía Internacional sobre la situación de los derechos humanos en el mundo ${ }^{65}$. Además, se ha dado cuenta que el mundo ha cambiado desde 2000 a la fecha y por tanto las ideas de paz, seguridad y justicia deben ser entendidas de manera diferente ${ }^{66}$.

\footnotetext{
S/2015/716 (2015), párrafos 28 a 32.

S/2015/716 (2015), párrafos 33 a 38.

S/2015/716 (2015), párrafos 4 y 5.

65 Amnistía Internacional, Informe Anual 2015/2016 de Amnistía Internacional sobre la situación de los derechos humanos en el mundo (2016), 14 a 18, http://amnistia.cl/web/wp-content/uploads/2016/02/Informe-2015-16-Amnist--aInternacional-1.pdf (consultado el 20 de junio de 2016).

66 Radhika Coomaraswamy, "A Global Study on the Implementation of United Nations Security Council resolution 1325”, Preventing conflict, transforming justice, securing the peace (2015), 13, http://wps.unwomen.org/ /media/files/un\%20women/wps/highlights/unw-global-study-1325-2015.pdf (consultado el 20 de junio de 2016).
} 
Así las cosas, del 2015 puede decirse que fue un año de inflexión en la evaluación de lo que han sido los resultados de la agenda mundial sobre mujeres, paz y seguridad, a la vez que el tiempo para desarrollar nuevas recomendaciones y reforzar las exhortaciones y llamados a los estados y la comunidad internacional en general a lograr cambios verdaderos, asumiendo como un hecho evidente que el escenario de hoy es muy diferente a aquel del año 2000, por lo cual las estrategias y acciones deben ser consecuentes con ello.

En ese contexto, en octubre de 2015 el Consejo de Seguridad adoptó la Resolución 2242 ${ }^{67}$, marcando con ello el inicio de una nueva etapa que crea y profundiza recomendaciones a partir del informe del Secretario General ${ }^{68}$ y exhorta a los estados, a partir de lo que han sido los primeros quince años de vida de la Resolución 1325 y de cara a la realidad internacional actual en materia de conflictos bélicos, internos o internacionalizados.

Uno de los aspectos relevantes de esta resolución es el llamado que hace a los estados que poseen un plan de acción nacional para la implementación de la Resolución 1325. Junto con reafirmar el empeño del CSNU de que se sigan aplicando plenamente y se refuercen entre sí esta resolución y las sucesoras, alienta a que se examinen las recomendaciones del Informe del Secretario General de septiembre de 2015 sobre las mujeres, y la paz y la seguridad, insta a que los estados evalúen las estrategias y recursos asignados para la implementación de la agenda sobre mujeres, paz y seguridad, y exhorta a los países que ya tienen plan de acción de acción nacional a que presenten información actualizada acerca de los progresos conseguidos en su implementación y examen durante los debates abiertos anuales del CSNU sobre mujeres, paz y seguridad $^{69}$.

Por su lado, el Secretario General ha dicho en 2015 que los planes constituyen una oportunidad para que los interesados nacionales identifiquen prioridades, determinen responsabilidades, asignen recursos y emprendan actividades en un plazo definido. Además, que existirían ciertos elementos comunes necesarios para que un plan de acción nacional pueda facilitar una acción coherente, selectiva y eficaz, como un fuerte liderazgo y una coordinación eficaz, procesos de diseño inclusivo, el cálculo de los costos y los presupuestos de los procesos de aplicación, supervisión y evaluación, y la flexibilidad para adaptarse a las nuevas situaciones $^{70}$.

Esto es especialmente importante para Chile que tiene en plena ejecución su Segundo Plan de Acción Nacional para la implementación de la Resolución del Consejo de Seguridad de la Organización de Naciones Unidas 1325/2000 "Mujeres, Seguridad y Paz"71. Al ser un país que

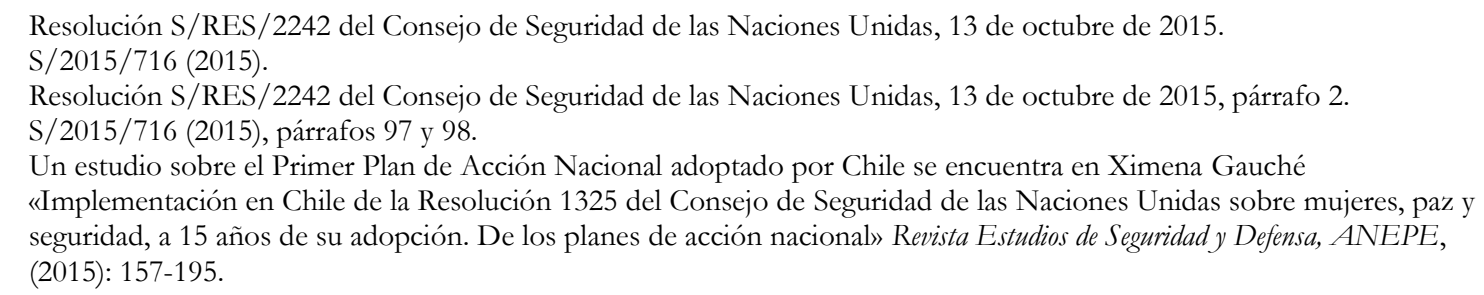


La Resolución 1325 del Consejo de Seguridad de las Naciones Unidas. A 15 años de su adopción Ximena Gauché Marchetti

contribuye en operaciones de paz... "tiene el imperativo de estar a la altura de los más altos estándares de equidad de género en el ámbito de la paz y la seguridad internacional”"72.

Conocer entonces la narrativa que las Naciones Unidas ha creado en el tema - a modo de un verdadero marco de vocación y acción normativa para los países como se ha ido poniendo en evidencia en este trabajo - así como asumir los cambios que la realidad manifiesta, estudiarlos y considerarlos en sus diferentes dimensiones de cara a seguir avanzando en equidad e inclusión de mujeres es un imperativo impuesto para nuestro país.

72 Chile, Segundo Plan de Acción nacional para la implementación de la resolución del Consejo de Seguridad de la Organización de las Naciones Unidas 1325/2000 “Mujeres, Seguridad y Paz", http:/ /www.gob.cl/wpcontent/uploads/2015/03/segundo-plan-de-accion-nacional.pdf (consultado el 20 de junio de 2016). 